\title{
Competitive Adsorption of Lubricant Base Oil and Ionic Liquid Additives at Air/Liquid and Solid/Liquid Interfaces
}

Dien $\mathrm{Ngo}^{1}$, Xin $\mathrm{He}^{2}$, Huimin $\mathrm{Luo}^{3}$, Jun $\mathrm{Qu}^{2}$, and Seong H. Kim${ }^{*}$

1. Department of Chemical Engineering and Materials Research Institute, Pennsylvania State University, University Park, PA, USA

2. Materials Science and Technology Division, Oak Ridge National Laboratory, Oak Ridge, TN, USA

3. Energy and Transportation Science Division, Oak Ridge National Laboratory, Oak Ridge, TN, USA

* Corresponding author: shk10@psu.edu

Number of pages: 6

Number of figures: 2

Number of tables: 4 


\section{Fittings of SFG spectra}

SFG spectra were fitted using the Lorentzian line shape described in the following equation. ${ }^{1}$

$$
I_{S F G}=\left|\sum_{m} \frac{A_{m}}{\omega_{m}-\omega_{I R}+i \Gamma_{m}}+n e^{-i \varepsilon}\right|^{2}
$$

In the above equation $A_{m}, \omega_{m}$, and $\Gamma_{m}$ are the amplitude (oscillator strength), wavenumber (frequency), and damping constant (line width), respectively, of the $m^{\text {th }}$ resonant mode. $\omega_{I R}$ is the tuning IR wavenumber (frequency). The parameters $n$ and $\varepsilon$ are the amplitude and phase of nonresonant background, respectively. The spectral deconvolution was performed using the Curve Fitting Tool in MATLAB. The fitting results are given in Tables S1-4. Note that the peak positions obtained from the fittings of the $2845-2930 \mathrm{~cm}^{-1}$ regions of $P P P$ spectra are somewhat less accurate due to low signal to noise ratio. 
Table S1. Fitting results of SSP spectra of n-hexadecane, PAO4, ionic liquids, and mixed liquids at air/liquid interface. $A_{m}, \omega_{m}$, and $\Gamma_{\mathrm{m}}$ are the amplitude, wavenumber, and damping constant, respectively, of the $m^{\text {th }}$ resonant mode. A $\equiv$ DEHP

\begin{tabular}{|c|c|c|c|c|c|c|c|c|c|c|c|c|c|c|c|}
\hline Sample & $A_{1}$ & $\begin{array}{c}\omega_{1} \\
\left(\mathbf{c m}^{-1}\right)\end{array}$ & $\begin{array}{c}\Gamma_{1} \\
\left(\mathbf{c m}^{-1}\right)\end{array}$ & $A_{2}$ & $\begin{array}{c}\omega_{2} \\
\left(\mathbf{c m}^{-1}\right)\end{array}$ & $\begin{array}{c}\Gamma_{2} \\
\left(\mathrm{~cm}^{-1}\right)\end{array}$ & $A_{3}$ & $\begin{array}{c}\omega_{3} \\
\left(\mathrm{~cm}^{-1}\right)\end{array}$ & $\begin{array}{c}\Gamma_{3} \\
\left(\mathbf{c m}^{-1}\right)\end{array}$ & $A_{4}$ & $\begin{array}{c}\omega_{4} \\
\left(\mathrm{~cm}^{-1}\right)\end{array}$ & $\begin{array}{c}\Gamma_{4} \\
\left(\mathrm{~cm}^{-1}\right)\end{array}$ & $A_{5}$ & $\begin{array}{c}\omega_{5} \\
\left(\mathbf{c m}^{-1}\right)\end{array}$ & $\begin{array}{c}\Gamma_{5} \\
\left(\mathbf{c m}^{-1}\right)\end{array}$ \\
\hline n-hexadecane & 3.76 & 2854 & 6.34 & 4.85 & 2875 & 7.90 & 1.59 & 2912 & 8.71 & 5.88 & 2925 & 10.76 & 4.52 & 2937 & 8.28 \\
\hline PAO4 & 3.32 & 2854 & 7.30 & 6.70 & 2875 & 8.50 & 4.26 & 2913 & 12.8 & 6.12 & 2928 & 10.35 & 3.65 & 2939 & 6.97 \\
\hline$[\mathrm{N888H}][\mathrm{A}]$ & 2.00 & 2857 & 9.86 & 5.21 & 2876 & 6.25 & - & - & - & 4.59 & 2921 & 16.90 & 4.47 & 2939 & 8.00 \\
\hline$[\mathbf{P 8 8 8 8}][\mathrm{A}]$ & 1.76 & 2856 & 8.34 & 5.42 & 2875 & 6.22 & - & - & - & 4.73 & 2922 & 15.21 & 3.79 & 2938 & 7.00 \\
\hline PAO4 + [N888H][A] & 2.93 & 2854 & 6.34 & 7.01 & 2875 & 8.72 & 3.33 & 2913 & 11.81 & 5.92 & 2928 & 10.31 & 3.58 & 2939 & 6.59 \\
\hline PAO4 + [P8888][A] & 3.31 & 2855 & 7.38 & 5.89 & 2876 & 7.95 & 2.48 & 2915 & 11.18 & 5.62 & 2929 & 10.26 & 3.95 & 2940 & 7.15 \\
\hline
\end{tabular}

Table S2. Fitting results of $P P P$ spectra of n-hexadecane, PAO4, ionic liquids, and mixed liquids at air/liquid interface. $A_{m}, \omega_{m}$, and $\Gamma_{m}$ are the amplitude, wavenumber, and damping constant, respectively, of the $m^{\text {th }}$ resonant mode. A $\equiv$ DEHP

\begin{tabular}{|c|c|c|c|c|c|c|c|c|c|c|c|c|c|c|c|}
\hline Sample & $A_{1}$ & $\begin{array}{c}\omega_{1} \\
\left(\mathrm{~cm}^{-1}\right)\end{array}$ & $\begin{array}{c}\Gamma_{1} \\
\left(\mathbf{c m}^{-1}\right)\end{array}$ & $A_{2}$ & $\begin{array}{c}\omega_{2} \\
\left(\mathbf{c m}^{-1}\right)\end{array}$ & $\begin{array}{c}\Gamma_{2} \\
\left(\mathrm{~cm}^{-1}\right)\end{array}$ & $A_{3}$ & $\begin{array}{c}\omega_{3} \\
\left(\mathrm{~cm}^{-1}\right)\end{array}$ & $\begin{array}{c}\Gamma_{3} \\
\left(\mathrm{~cm}^{-1}\right)\end{array}$ & $A_{4}$ & $\begin{array}{c}\omega_{4} \\
\left(\mathbf{c m}^{-1}\right)\end{array}$ & $\begin{array}{c}\Gamma_{4} \\
\left(\mathbf{c m}^{-1}\right)\end{array}$ & $A_{5}$ & $\begin{array}{c}\omega_{5} \\
\left(\mathrm{~cm}^{-1}\right)\end{array}$ & $\begin{array}{c}\Gamma_{5} \\
\left(\mathbf{c m}^{-1}\right)\end{array}$ \\
\hline n-hexadecane & 0.61 & 2857 & 5.49 & 0.40 & 2880 & 2.24 & 4.04 & 2899 & 15.56 & 6.35 & 2922 & 12.38 & 8.74 & 2963 & 9.33 \\
\hline PAO4 & 0.56 & 2856 & 6.20 & 0.40 & 2879 & 1.98 & 3.22 & 2896 & 13.95 & 4.92 & 2921 & 12.94 & 8.84 & 2963 & 9.33 \\
\hline$[\mathbf{N 8 8 8 H}][\mathrm{A}]$ & 0.27 & 2861 & 4.95 & 0.69 & 2880 & 4.00 & 1.08 & 2898 & 8.69 & 3.41 & 2920 & 14.35 & 7.65 & 2965 & 7.80 \\
\hline [P8888][A] & 0.05 & 2860 & 3.01 & 0.40 & 2879 & 3.00 & 1.71 & 2905 & 13.06 & 2.15 & 2924 & 11.25 & 7.81 & 2963 & 7.84 \\
\hline PAO4 + [N888H][A] & 0.53 & 2858 & 5.44 & 0.51 & 2879 & 3.01 & 3.66 & 2900 & 14.95 & 4.00 & 2922 & 12.26 & 8.91 & 2963 & 9.36 \\
\hline PAO4 + [P8888][A] & 0.54 & 2858 & 5.86 & 0.49 & 2880 & 3.00 & 3.66 & 2902 & 16.55 & 3.98 & 2924 & 11.51 & 9.66 & 2964 & 9.47 \\
\hline
\end{tabular}


Table S3. Fitting results of SSP spectra of n-hexadecane, PAO4, ionic liquids, and mixed liquids at silica/liquid interface. $A_{m}, \omega_{m}$, and $\Gamma_{m}$ are the amplitude, wavenumber, and damping constant, respectively, of the $m^{\text {th }}$ resonant mode. A $\equiv \mathrm{DEHP}$

\begin{tabular}{|c|c|c|c|c|c|c|c|c|c|c|c|c|c|c|c|}
\hline Sample & $A_{1}$ & $\begin{array}{c}\omega_{1} \\
\left(\mathrm{~cm}^{-1}\right)\end{array}$ & $\begin{array}{c}\Gamma_{1} \\
\left(\mathbf{c m}^{-1}\right)\end{array}$ & $A_{2}$ & $\begin{array}{c}\omega_{2} \\
\left(\mathbf{c m}^{-1}\right)\end{array}$ & $\begin{array}{c}\Gamma_{2} \\
\left(\mathbf{c m}^{-1}\right)\end{array}$ & $A_{3}$ & $\begin{array}{c}\omega_{3} \\
\left(\mathrm{~cm}^{-1}\right)\end{array}$ & $\begin{array}{c}\Gamma_{3} \\
\left(\mathbf{c m}^{-1}\right)\end{array}$ & $A_{4}$ & $\begin{array}{c}\omega_{4} \\
\left(\mathbf{c m}^{-1}\right)\end{array}$ & $\begin{array}{c}\Gamma_{4} \\
\left(\mathbf{c m}^{-1}\right)\end{array}$ & $A_{5}$ & $\begin{array}{c}\omega_{5} \\
\left(\mathbf{c m}^{-1}\right)\end{array}$ & $\begin{array}{c}\Gamma_{5} \\
\left(\mathbf{c m}^{-1}\right)\end{array}$ \\
\hline n-hexadecane & 3.67 & 2852 & 8.83 & 0.81 & 2868 & 5.00 & 12.42 & 2919 & 13.68 & 1.82 & 2929 & 5.86 & 1.58 & 2954 & 7.45 \\
\hline PAO4 & 1.85 & 2855 & 5.00 & 4.23 & 2869 & 8.38 & 3.11 & 2911 & 10.93 & 6.12 & 2925 & 10.64 & 7.11 & 2942 & 10.05 \\
\hline [N888H][A] & 1.04 & 2849 & 5.00 & 2.51 & 2873 & 5.24 & 5.44 & 2904 & 23.61 & - & - & - & 12.39 & 2943 & 14.15 \\
\hline [P8888][A] & 1.61 & 2847 & 5.43 & 2.41 & 2873 & 6.20 & 2.88 & 2915 & 11.06 & - & - & - & 12.89 & 2941 & 14.12 \\
\hline PAO4 + [N888H][A] & - & - & - & 2.10 & 2877 & 5.29 & 3.80 & 2891 & 10.52 & 5.61 & 2913 & 11.14 & 9.11 & 2943 & 11.12 \\
\hline PAO4 + [P8888][A] & 1.00 & 2844 & 3.00 & 4.04 & 2873 & 7.23 & 6.62 & 2914 & 12.35 & - & - & - & 10.18 & 2941 & 12.21 \\
\hline
\end{tabular}

Table S4. Fitting results of $P P P$ spectra of n-hexadecane, PAO4, ionic liquids, and mixed liquids at silica/liquid interface. $A_{m}, \omega_{m}$, and $\Gamma_{m}$ are the amplitude, wavenumber, and damping constant, respectively, of the $m^{\text {th }}$ resonant mode. A DEHP

\begin{tabular}{|c|c|c|c|c|c|c|c|c|c|c|c|c|c|c|c|}
\hline Sample & $A_{1}$ & $\begin{array}{c}\omega_{1} \\
\left(\mathrm{~cm}^{-1}\right)\end{array}$ & $\begin{array}{c}\Gamma_{1} \\
\left(\mathbf{c m}^{-1}\right)\end{array}$ & $A_{2}$ & $\begin{array}{c}\omega_{2} \\
\left(\mathrm{~cm}^{-1}\right)\end{array}$ & $\begin{array}{c}\Gamma_{2} \\
\left(\mathrm{~cm}^{-1}\right)\end{array}$ & $A_{3}$ & $\begin{array}{c}\omega_{3} \\
\left(\mathrm{~cm}^{-1}\right)\end{array}$ & $\begin{array}{c}\Gamma_{3} \\
\left(\mathbf{c m}^{-1}\right)\end{array}$ & $A_{4}$ & $\begin{array}{c}\omega_{4} \\
\left(\mathbf{c m}^{-1}\right)\end{array}$ & $\begin{array}{c}\Gamma_{4} \\
\left(\mathrm{~cm}^{-1}\right)\end{array}$ & $A_{5}$ & $\begin{array}{c}\omega_{5} \\
\left(\mathrm{~cm}^{-1}\right)\end{array}$ & $\begin{array}{c}\Gamma_{5} \\
\left(\mathbf{c m}^{-1}\right)\end{array}$ \\
\hline n-hexadecane & 0.10 & 2860 & 5.00 & 1.41 & 2870 & 5.57 & 9.27 & 2892 & 18.21 & 5.30 & 2948 & 10.28 & - & - & - \\
\hline PAO4 & - & - & - & 0.65 & 2878 & 4.00 & 1.21 & 2897 & 5.00 & 2.38 & 2915 & 8.17 & 6.57 & 2960 & 7.32 \\
\hline [N888H][A] & 0.89 & 2844 & 6.87 & 1.56 & 2871 & 6.59 & - & - & - & 0.14 & 2922 & 5.00 & 4.37 & 2941 & 11.00 \\
\hline [P8888][A] & 0.84 & 2849 & 3.10 & 1.84 & 2872 & 5.00 & - & - & - & - & - & - & 12.26 & 2941 & 12.33 \\
\hline PAO4 + [N888H][A] & 0.52 & 2849 & 3.00 & 3.19 & 2874 & 6.82 & - & - & - & - & - & - & 13.54 & 2942 & 13.79 \\
\hline PAO4 + [P8888][A] & 0.47 & 2844 & 2.00 & 2.50 & 2873 & 5.83 & - & - & - & - & - & - & 13.38 & 2943 & 13.13 \\
\hline
\end{tabular}



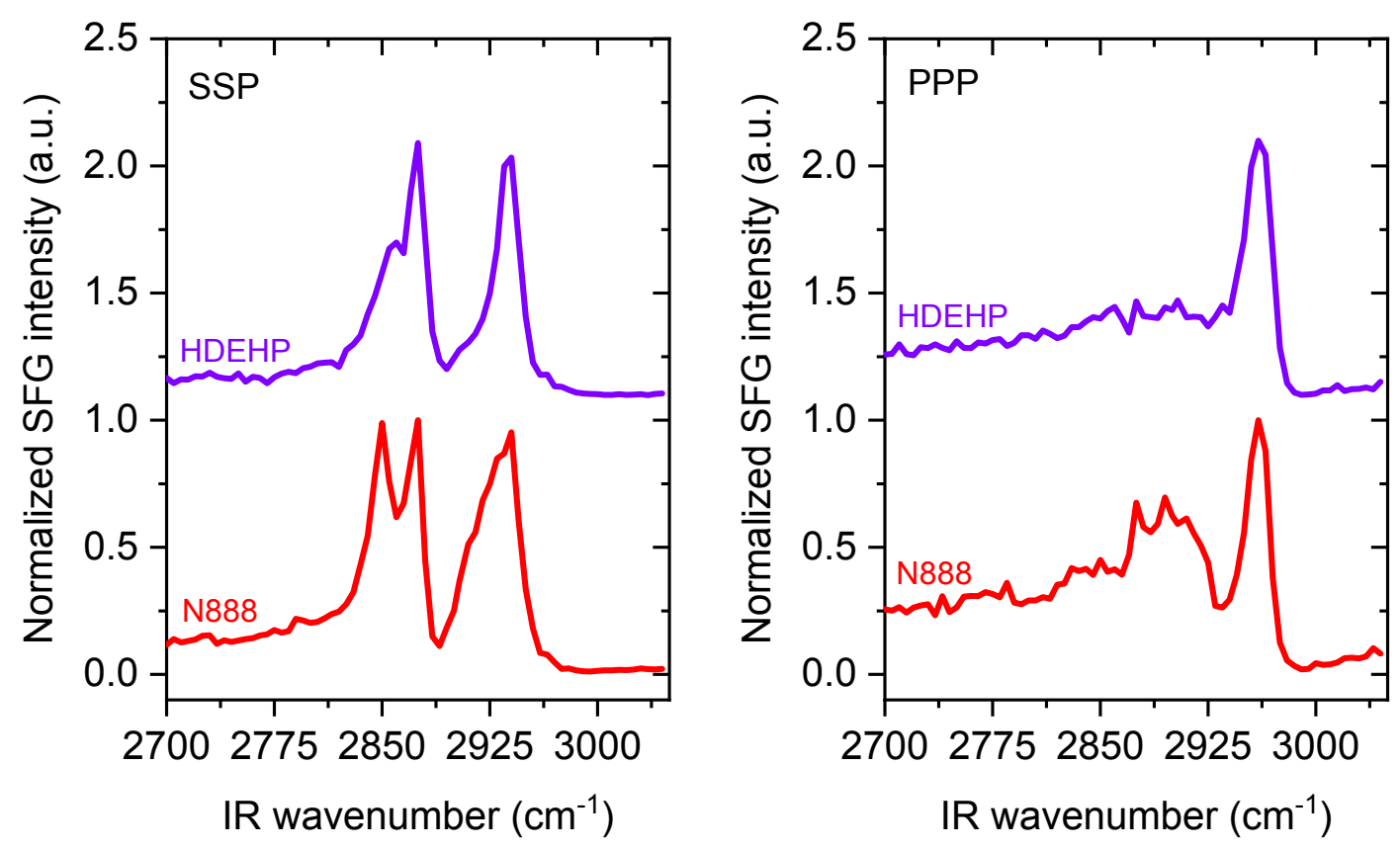

Figure S1. SFG spectra of N888 and HDEHP at air/liquid interface.
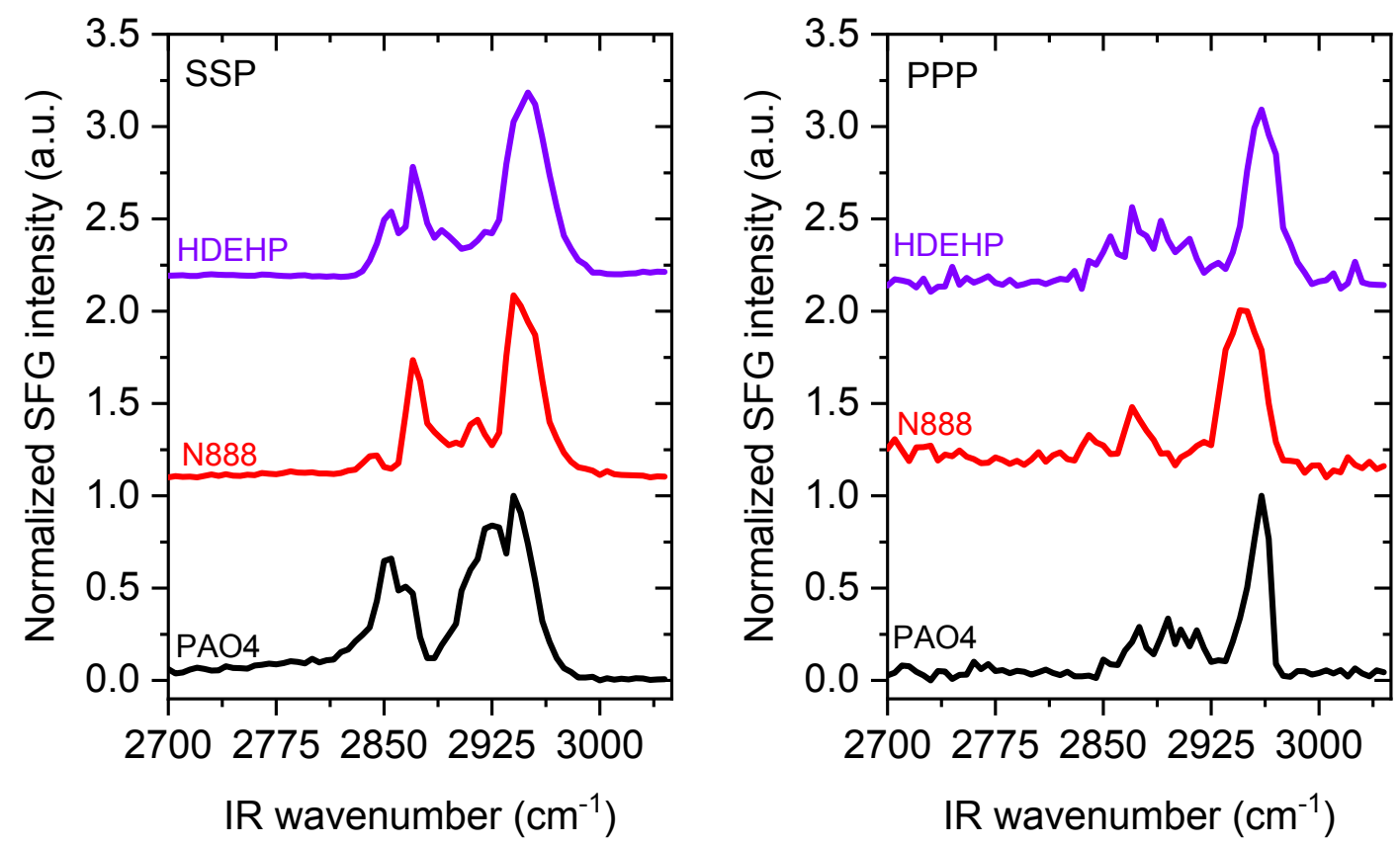

Figure S2. SFG spectra of PAO4, N888, and HDEHP at the silica surface; A $\equiv$ DEHP. 


\section{References}

1. Ngo, D.; Liu, H.; Chen, Z.; Kaya, H.; Zimudzi, T. J.; Gin, S.; Mahadevan, T.; Du, J.; Kim, S. H., Hydrogen bonding interactions of $\mathrm{H} 2 \mathrm{O}$ and $\mathrm{SiOH}$ on a boroaluminosilicate glass corroded in aqueous solution. npj Materials Degradation 2020, 4 (1), 1. 